\title{
NLRP3 inflammasome couples purinergic signaling with activation of the complement cascade for the optimal release of cells from bone marrow
}

\author{
Mariusz Z. Ratajczak ${ }^{1,2} \cdot$ Mateusz Adamiak $^{2} \cdot$ Arjun Thapa $^{1} \cdot$ Kamila Bujko $^{1} \cdot$ Katarzyna Brzezniakiewicz-Janus $^{3}$. \\ Anna M. Lenkiewicz ${ }^{2}$
}

Received: 21 January 2019 / Revised: 17 February 2019 / Accepted: 20 February 2019 / Published online: 7 March 2019

(c) The Author(s) 2019. This article is published with open access

\begin{abstract}
The mechanisms that regulate egress of hematopoietic stem/progenitor cells (HSPCs) into peripheral blood (PB) in response to stress, inflammation, tissue/organ injury, or administration of mobilization-inducing drugs are still not well understood, and because of the importance of stem cell trafficking in maintaining organism homeostasis, several complementary pathways are believed to be involved. Our group proposes that mobilization of HSPCs is mainly a result of sterile inflammation in the bone marrow (BM) microenvironment in response to pro-mobilizing stimuli and that during the initiation phase of the mobilization process BM-residing cells belonging to the innate immunity system, including granulocytes and monocytes, release danger-associated molecular pattern molecules (DAMPs, also known as alarmins), reactive oxygen species (ROS), as well as proteolytic and lipolytic enzymes. These factors together orchestrate the release of HSPCs into PB. One of the most important DAMPs released in the initiation phase of mobilization is extracellular adenosine triphosphate, a potent activator of the inflammasome. As a result of its activation, IL-1 $\beta$ and IL-18 as well as other promobilizing mediators, including DAMPs such as high molecular group box 1 (Hmgb1) and S100 calcium-binding protein A9 (S100a9), are released. These DAMPs are important activators of the complement cascade (ComC) in the mannanbinding lectin (MBL)-dependent pathway. Specifically, Hmgb1 and S100a9 bind to MBL, which leads to activation of MBL-associated proteases, which activate the ComC and in parallel also trigger activation of the coagulation cascade (CoaC). In this review, we will highlight the novel role of the innate immunity cell-expressed NLRP3 inflammasome, which, during the initiation phase of HSPC mobilization, couples purinergic signaling with the MBL-dependent pathway of the ComC and, in parallel, the CoaC for optimal release of HSPCs. These data are important to optimize the pharmacological mobilization of HSPCs.
\end{abstract}

\section{Introduction}

Clinical data indicate that a significant number of donors of hematopoietic stem/progenitor cells (HSPCs) respond

Mariusz Z. Ratajczak

mzrata01@louisville.edu

1 Stem Cell Institute at James Graham Brown Cancer Center, University of Louisville, Louisville, KY, USA

2 Center for Preclinical Studies and Technology, Department of Regenerative Medicine Warsaw Medical University, Warsaw, Poland

3 Department of Hematology, University of Zielona Gora, Hospital Gorzow Wlkp, Zielona Gora, Poland poorly to mobilization by granulocyte colony-stimulating factor (G-CSF) and CXCR4-blocking small-molecule AMD3100 [1-5]. Therefore, the optimization of stem cell mobilization and the subsequent enhancement of seeding efficiency of HSPCs in BM after transplantation are important goals to improve clinical outcomes of transplantations, and this will be addressed in this review. We will employ in this review for reasons of simplicity the term HSPCs as both stem and progenitor cells are present in the mobilization product; however mechanisms described herein may affect hematopoietic stem cells (HSCs) and hematopoietic progenitor cells (HPCs) in various ways. This, however, requires further studies.

It is well known that HSPCs are nonstop travelers throughout the body. During embryogenesis, they migrate between different organs that maintain active 
hematopoiesis, and later on in postnatal life, a small number continue to circulate in peripheral blood (PB) [1-5]. These HSPCs circulating under steady-state conditions patrol tissues and organs in search of damage and keep in balance the pool of stem cells, which is spread throughout the hematopoietic niches in remote areas of bone marrow (BM) tissue [1, 5-7]. This circulation of HSPCs undergoes circadian rhythm changes, in which more of these cells are present in PB in the early morning hours than late at night [8]. We have proposed that this process is related, at least partially, to changes in tonus of the complement cascade (ComC) and the coagulation cascade $(\mathrm{CoaC})$, which are regulated by deep-sleep hypoxia $[1,9]$.

As is well known, HSPCs are retained in their BM niches by signals involving the interactions between (i) the $\alpha$ chemokine receptor CXCR 4 and (ii) an $\alpha 4 \beta 1$ integrin dimer composed of CD49d (alpha 4) and CD29 (beta 1), also known as very-late antigen 4 (VLA-4), present on the HSPC surface [7, 10, 11]. Both of these cell surface receptors, CXCR4 and VLA-4, interact with the corresponding ligands, stromal-derived factor 1 (SDF-1) and vascular cell-adhesion molecule 1 (VCAM-1), respectively, which are expressed by the cellular components of stem cell niches $[1,5,7]$.

Pharmacological mobilization is a means to obtain HSPCs for hematopoietic transplantation and is induced by pro-mobilizing drugs. During administration of these drugs (e.g., G-CSF or AMD3100) the number of these cells in PB may increase up to 100 -fold over steady-state conditions [1-7]. Mobilized HSPCs are subsequently harvested from PB by leukapheresis. The exact mechanisms that regulate this massive egress of HSPCs from BM into PB are still not well understood, and most likely several complementary mechanisms are involved [1-5, 12]. Our group proposes that pharmacological mobilization of HSPCs occurs as a result of "sterile" inflammation that is induced in the BM microenvironment in response to pro-mobilizing stimuli $[1,13,14]$. According to the definition, sterile inflammation is an inflammatory process that occurs in the absence of any microorganisms and during pharmacological mobilization is triggered by G-CSF or AMD3100 [15]. Sterile inflammation, like microbial-induced inflammation, is initiated by the activation of cellular elements of innate immunity, including neutrophils and macrophages, as well as by activation of the ComC [15].

Overall, we can divide the HSPC mobilization process into three principal phases: (i) initiation, (ii) amplification, and (iii) execution. In the (first) initiation phase, promobilizing stimuli activate the cellular innate immunity network by stimulating BM-residing granulocytes and monocytes and perhaps also other innate immunity cells in the BM microenvironment to release danger-associated molecular pattern molecules (DAMPs), reactive oxygen species (ROS), and proteolytic and lipolytic enzymes [1, 3, 14-18]. As we recently demonstrated, an important role is also played by the activation of purinergic signaling, involving release of extracellular nucleotides (EXNs) from the activated cells [19]. The most important member of this class of molecules is adenosine triphosphate (ATP), a potent mediator in the extracellular purinergic signaling network [20]. These complementary signals allow HSPC detachment and egress from BM stem cell niches during the initiation phase of mobilization. What will be addressed in more details later on in this review is that the initiation phase leads to the activation of the ComC by triggering mannanbinding lectin (MBL)-dependent pathway of ComC activation. Next, during the amplification phase of mobilization, ComC becomes additionally activated by alternative activation pathway. Finally, in the execution phase of mobilization ComC final cleavage fragments $\mathrm{C} 5$ and $\mathrm{C} 5 \mathrm{a}$ as well as activated $\mathrm{CoaC}$ are required for the optimal egress of HSPCs from BM into PB [14, 19].

In this review, we will present evidence from our recent investigations that during the initiation phase of mobilization, the ATP-activated inflammasome NLRP3 has an important role in activation of the ComC and CoaC. As depicted in Fig. 1a, the inflammasome has the role of a gear or cogwheel that couples activated purinergic signaling with activation of the ComC and $\mathrm{CoaC}$. We will discuss this novel concept about the role of inflammasomes in the mobilization of HSPCs, as modulation of inflammasome activity may become an important means to improving HSPC yield during clinical pharmacological mobilization. The inflammasomes may also be involved in the egress of HSPCs into PB during other situations, such as systemic inflammation or stress related to tissue/organ injury, and it may also have a role in the circadian circulation of HSPCs in PB [21-23].

\section{The inflammasomes as important part of the innate immunity network}

Inflammasomes are multiprotein oligomer complexes and important components of the innate immunity network that are triggered during "sterile" inflammation in response to DAMPs (also known as alarmins), which are released by activated or damaged cells, and during infections in response to pathogen-associated molecular pattern molecules (PAMPs) [21-23]. Here we will focus on the NLRP3 member of this family, which is highly expressed in innate immunity cells $[21,22]$.

The NLRP3 inflammasome is expressed by myeloid cells, including Gr- $1^{+}$granulocytes and monocytes, and is currently the best-characterized member of this family operating in hematopoietic cells [21, 22]. It is composed of several proteins, including NLRP3, CARD-containing 
A

Fig. 1 a The inflammasome as a gear or cogwheel that couples purinergic signaling with the complement cascade (ComC) in the process of HSPC mobilization. Pro-mobilizing stimuli (e.g., G-CSF) (1) release ATP from activated innate immunity $\mathrm{Gr}-1^{+}$cells $(2)$, which activates intracellular inflammasome via $\mathrm{P} 2 \mathrm{X} 7$ purinergic receptors (3). As result of inflammasome activation several DAMPs are released, including Hmgb1 and S100a9 (4), which are recognized by MBL and activate the ComC in the mannan-binding lectin-dependent pathway. Activation of the ComC leads to release of $\mathrm{C} 5$ cleavage fragments that are crucial for optimal release of HSPCs from BM into PB (5). In parallel, what is not shown in this scheme, the CoaC is also activated

adaptor (ASC), and caspase 1, and is responsible for the activation of inflammatory responses (Fig. 1b) [23]. Activation of intracellular caspase 1 promotes maturation and secretion of pro-inflammatory cytokines, such as interleukin $1 \beta$ (IL-1 $\beta$ ) and interleukin 18 (IL-18), which are activated inside cells by caspase 1 proteolytic cleavage of their proforms (pro-IL-1 $\beta$ and pro-IL-18) before release into the extracellular space [21-23]. This is facilitated by the creation of cell membrane pores owing to insertion into the membrane another caspase 1 cleavage product, $\mathrm{N}$-gasdermin, that is, gasdermin protein fragment (Fig. 1b) [21-23].

It is known that the interleukin 1 family is composed of a group of 11 members, which include a complex network of pro-inflammatory cytokines that regulate and initiate inflammatory responses [24]. IL-1 $\beta$, together with IL- $1 \alpha$, is the most-studied member of this cytokine family. In addition to granulocytes, monocytes, and macrophages, it is also secreted by dendritic cells, B lymphocytes, natural killer cells, microglia, fibroblasts, and epithelial cells [24]. IL-1 $\beta$ is synthesized after stimulation by DAMPs (e.g., ATP, Hmgb1, and S1000a9) and PAMPs (e.g., liposaccharideLPS). In contrast to IL- $1 \alpha$, its expression is induced by the transcription factor NF- $\mathrm{KB}$. Another member of the IL-1 family, IL-18, is also secreted as part of inflammasome

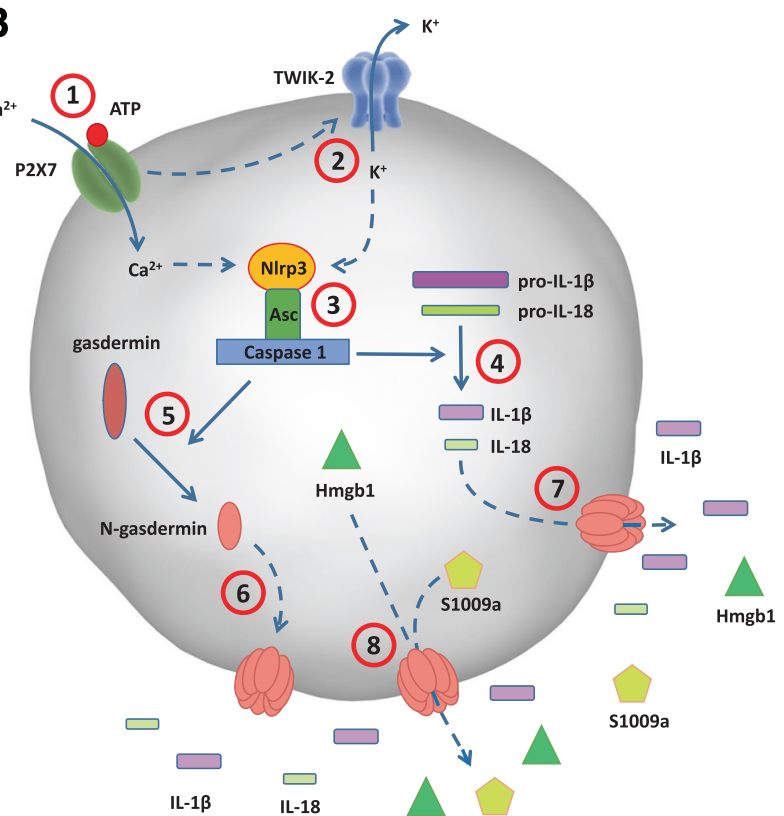

in an MBL-dependent manner. b The most important steps in the intracellular activation of NRLP3 inflammasome by ATP: Extracellular ATP activates P2X7 (step 1), which subsequently activates $\mathrm{K}^{+}$ efflux channel TWIK2 (step 2). A decrease in $\mathrm{K}^{+}$intracellular levels triggers the activation of the NRLP3 inflammasome complex (step 3). In response to this caspase, 1 cleaves pro-IL-1 $\beta$ and IL-18 to active ready for secretion IL-1 $\beta$ and IL-18 (step 4), and in addition cleaves gasdermin that releases $N$-gasdermin (Step 5 ) that insert into the cell membrane to create pores (step 6) for the release of IL-1 $\beta$ and IL-18 (step 7) as well as DAMPs (step 8)

activation by innate immunity cells. It has been reported that both IL-1 $\beta$ and IL-18 induce leukocytosis when injected into mice [25]. Besides pro-inflammatory properties both cytokines exert several other pleiotropic effects in hematopoiesis. For example, IL-1 $\beta$ may radioprotect adult BM HSPCs and increase their proliferation and differentiation [25]. On the other hand, IL-18 stimulates the secretion of several hematopoietic modulators including interferon- $\gamma$, IL-6, and granulocyte-macrophage colonystimulating factor.

It is widely accepted that release of active IL-1 $\beta$ and IL18 from cells is one piece of molecular evidence for activation of the inflammasome [21, 22]. However, in addition to these interleukins, upon activation of the inflammasome innate immunity cells release several other DAMPs, including Hmgb1 and S100a9, which are recognized by the PB-circulating collagen-containing C-type lectin (collectin) known as MBL, an important soluble member of the group of pattern-recognition receptors. Innate immunity cells also release ROS that expose neoepitopes on the surface of cells in the BM microenvironment, which after binding with naturally occurring IgM antibodies, also become targets for MBL [26]. ROS alone may also trigger activation of the inflammasome in innate immunity cells [21-23]. Based on 
these mechanisms, the NLRP3 inflammasome activates the MBL-dependent pathway of the ComC by cell-secreted DAMPs (e.g., Hmgb1 or S100a9) as well as ROS and also collaterally triggers another ancient proteolytic cascade, the CoaC. It is important to state, which in addition to NLRP3 inflammasome, other important members of this family including NLRP1 and NLRP12, which are also highly expressed in hematopoietic cells and respond to DAMPs $[27,28]$ may play similar role. Moreover, similarly, the potential involvement of NLRC4 and AIM2 inflammasomes in "sterile" inflammation of BM requires further studies [29].

Using appropriate knockout (KO) mice, we previously demonstrated that of the three pathways of ComC activation, (i) the C1q-mediated classical pathway, (ii) the MBLmediated pathway, and (iii) the factor $\mathrm{B}(\mathrm{FB})$-regulated alternative pathway, the MBL pathway of the ComC has the most important role in pharmacological mobilization of HSPCs [26]. In support of this conclusion, MBL-KO mice, which have a defective MBL pathway of ComC activation, and not $\mathrm{Clq}-\mathrm{KO}$ mice, which do not activate the classical ComC pathway, are poor mobilizers [1, 30]. Our most current research also indicates involvement of the alternative ComC pathway, which, under steady-state conditions, is continuously auto-activated at a low level in PB and potentiates MBL-mediated activation of the ComC during mobilization. Specifically, FB-KO mice, which do not activate the alternative pathway of the ComC are, like MBL-KO mice, poor mobilizers (manuscript in preparation). This finding supports an active involvement of the alternative pathway of ComC activation during the amplification phase of the mobilization process.

The involvement of the NLRP3 inflammasome in mobilization of HSPCs is supported by our recent results, demonstrating that mice exposed to MCC950 inhibitor of the ASC that is a crucial component of NRLP3 inflammasome [21-23] become poor mobilizers (manuscript in preparation). What is also important to emphasize that, in addition to Hgmb1 and ROS released from myeloid cells owing to inflammasome activation, IL-1 $\beta$ and IL-18 released from innate immunity cells during inflammasome activation also increase on their own the egress of cells from BM. Both of these cytokines are strong pro-mobilizing mediators, and to corroborate this we confirmed the promobilizing activity of IL-1 $\beta$ [25] and determined that another inflammasome-released cytokine, IL-18, is also a potent pro-mobilizing factor. Interestingly, although neither IL-1 $\beta$ nor IL-18 alone can chemoattract HSPCs, mice injected with these cytokines release a comparable number of HSPCs from BM as the administration of AMD3100. At this point, the molecular mechanism behind this phenomenon is unclear, but we propose that secreted IL-1 $\beta$ and IL18, by means of an autocrine positive-feedback loop, potentiate activation of the NLRP3 inflammasome to release more Hmgb1 and ROS and thus amplify the MBL-mediated pathway of ComC activation. Supporting such a mechanism is the finding that IL-1 $\beta$ and IL-18 receptors are highly expressed by innate immunity cells.

It is well known that activation of the NLRP3 inflammasome requires two signals [21-23]. The first is a priming signal that is mediated by bacterial LPS activating Toll-like receptor 4 or by the TNF- $\alpha$-activating TNF receptor. This first signal induces the NF- $\kappa \mathrm{B}$ pathway, leading to upregulation of pro-IL-1 $\beta$ and NLRP3 protein levels and can also be amplified by autocrine-secreted IL- $1 \beta$ or IL-18 [21-23]. The second signal promotes the assembly of CARD-containing adaptor protein (ASC) and pro-caspase 1, leading to activation of the NLRP3 inflammasome complex [22]. What is important for the topic of this review, this second signal under noninfectious conditions, as seen for example during sterile inflammation, is provided by extracellular ATP, which leads to inflammasome activation by activating $\mathrm{P} 2 \mathrm{X} 7$ purinergic receptors on the surface of innate immunity cells and thereby triggering potassium efflux [21-23]. Recently, an important role of the potassium efflux channel TWIK2 has been found in activation of the NLRP3 inflammasome [31].

In further support of a role for the inflammasome in the mobilization process, LPS, which provides the first priming signal for inflammasome activation, has previously been demonstrated in mice to facilitate the mobilization process, as mice exposed to antibiotics that remove LPS-producing Gram-negative bacteria form the intestine show a decrease in the number of mobilized HSPCs [32, 33]. On the other hand, defective release of ATP by pannexin [19] or connexin [34] channels or defective release in P2X7-KO mice, which do not respond to the ATP-mediated second signal, both result in poor mobilization in response to G-CSF. This result may be explained at the molecular level by the fact that ATP-P2X7 signaling opens the inflammasometriggering TWIK2 potassium efflux channel [31]. What is also important is that activation of ComC activates NRLP3 and most likely NRLP1 inflammasome by direct stimulation of $\mathrm{C} 3 \mathrm{a}$ and $\mathrm{C} 5 \mathrm{a}$ receptors on innate immunity cells by $\mathrm{C} 5 \mathrm{a}$ by $\mathrm{C} 3$ and $\mathrm{C} 5$ cleavage products as well as owing to increasing cell membrane permeability for potassium efflux by final mediator of ComC activation that is $\mathrm{C} 5 \mathrm{bC} 9$ membrane attack complex (MAC) [21, 22]. This further support a crucial involvement of ComC activation as orchestrator of HSPCs mobilization [1, 11].

\section{ATP as a crucial mediator of purinergic signaling, activating the NLRP3 inflammasome}

As is well known, primarily, intracellular ATP provides energy to drive many processes in living cells and is 
involved in energy transfer. In addition, when secreted from the cells, it becomes a crucial mediator in the extracellular purinergic signaling network [35]. Purinergic signaling is considered to be an evolutionarily ancient signaling mechanism that regulates several aspects of cell biology, including providing chemotactic signals and modulating the responsiveness of innate and acquired immune cells to inflammatory cues $[19,20]$. Extracellular ATP binds to P2 purinergic receptors expressed on all cell types, including hematopoietic cells [35]. Based on their structural characteristics, P2 purinergic receptors are subdivided into metabotropic $(\mathrm{P} 2 \mathrm{Y})$ and ionotropic channel $(\mathrm{P} 2 \mathrm{X})$ receptors. The $\mathrm{P} 2 \mathrm{Y}$ receptor family includes eight $\mathrm{G}$ proteincoupled receptors (P2Y1, 2, 4, 6, 11, 12, 13, and 14). By contrast, the $\mathrm{P} 2 \mathrm{X}$ receptor family consists of seven ionotropic channel members (P2X1, 2, 3, 4, 5, 6, and 7), which, after stimulation by ATP, allow for efflux of $\mathrm{K}^{+}$and influx of $\mathrm{Ca}^{2+}$ and $\mathrm{Na}^{+}$. The P2X7 receptor, which has a role in ATP-mediated NRLP3 inflammasome activation, belongs to this second family of receptors [35].

ATP, a major ligand for $\mathrm{P} 2$ receptors, is released from the cells by exocytosis or in a conductive mechanism that involves two types of plasma membrane maxi ion channels or pore-forming channels, including (i) pannexins and (ii) connexins [35]. Pannexins predominantly exist as large transmembrane channels connecting the intracellular and extracellular space, allowing the passage of ions and small molecules, including ATP, between these compartments. We have shown that inhibition of pannexin 1 with probenecid or pannexin blocking peptides [19] results in a decrease in ATP release from BM-residing innate immunity cells and, as a consequence, impairs G-CSF and CXCR4 antagonist (AMD3100)-mediated mobilization of HSPCs [19]. Like pannexins, certain connexins (e.g., Cx43) that are located to non-junctional regions of the cell membrane may also release ATP into the extracellular space. Supporting the role of CX43 connexin in extracellular release of ATP, it has been reported in an excellent paper that $\mathrm{Cx} 43-\mathrm{KO}$ mice are poor mobilizers [34].

Based on these findings, ATP secreted from G-CSFactivated neutrophils and monocytes as a DAMP molecule is involved in the induction of sterile inflammation and couples the purinergic signaling network with the ComC via the NLRP3 inflammasome (Fig. 1). Most important is its interaction with the $\mathrm{P} 2 \mathrm{X} 7$ receptor on the surface of cells of the innate immunity system [19]. Interestingly, human loss or gain of function of P2X7 expression is differently influenced by the inheritance of various P2X7 singlenucleotide polymorphisms (SNPs). It has been reported that the Ala348Thr polymorphism results in a threefold increase in P2X7 activity over wild type [36]. Moreover, when Ala348Thr is co-inherited with Gln460Arg as a haplotype, the activity of P2X7 is further amplified to fivefold over those subjects not carrying this haplotype. In a recent report, it was demonstrated that the presence of the SNP Gln460Arg co-inherited with Ala348Thr, which leads to P2X7 hyperactivity, results in a significant increase in HSPC mobilization [36]. This result supports the important role of the ATP-P2X7-NLRP3 inflammasome axis in this process.

ATP, however, also activates several P2Y G proteincoupled purinergic receptors in parallel, and the exact involvement of these receptors in HSPC mobilization requires further study [37]. Nevertheless, a significant role seems to be played here by ATP-mediated upregulation of phospholipase $\beta 2$ (PLC- $\beta 2$ ) in hematopoietic cells [37]. Specifically, PLC- $\beta 2$, which is primarily involved in the intracellular signaling cascade, when released from activated granulocytes during degranulation digests the glycolipid glycosylphosphatidylinositol anchor, which plays a crucial role in maintaining the integrity of lipid rafts on HSPC extracellular membranes [1, 11]. As mentioned above, lipid rafts contain CXCR4 and VLA-4 receptors, which are crucial for anchoring HSPCs to their respective ligands SDF-1 and VCAM-1 in their BM niches [1-5, 11]. Given this mechanism, ATP involvement in the initial phase of mobilization of HSPCs is based both on activation of the inflammasome and on the parallel release of PLC- $\beta 2$ from neutrophils [37]. The importance of granulocytes in the mobilization process has been reported in the past and can be understood [38] in light of recent observations by their (i) involvement in triggering inflammasome activation and (ii) the release of proteolytic and lipolytic enzymes [21, 22, 39].

In summary, activation of the NLRP3 inflammasome releases (i) IL-1 $\beta$ and IL-18, which results in its autoactivation by an autocrine positive-feedback loop, and (ii) several DAMPs, such as Hmgb1 and S100a9, which are recognized by MBL circulating in PB. DAMP-MBL complexes activate mannan-associated proteases (MASPs), which cleave/activate both $\mathrm{C} 3$ and prothrombin, which in turn induce activation of the ComC and CoaC [26]. Of note, the levels of IL-1 $\beta$, IL-18, Hmgb1, and S100a9 increase in biological fluids during sterile inflammation, and this is seen during HSPC mobilization. Fig. 2 demonstrates induced expression of mRNA encoding Nlrp3, Casp 1, and IL-1 $\beta$ in $\mathrm{PB}$ mononuclear cells in mice mobilized for 3 days by GCSF.

We have also identified in the past two important inhibitors of HSPC mobilization: (i) heme oxygenase 1 (HO-1) [40] and (ii) inducible nitric oxide synthase (iNOS) [41] (Fig. 2). Both of these enzymes have anti-inflammatory activity, and both inhibit release of HSPCs from BM into $\mathrm{PB}$. What is important for the topic of this review, both HO1 and iNOS have been reported to be NLRP3 inflammasome inhibitors [42-44]. 
Fig. 2 Molecular evidence of inflammasome activation in response to pro-mobilizing agents. Expression of Nlrp3 a, Casp $1 \mathbf{b}$, and IL- $1 \beta \mathbf{c}$ genes in mice peripheral blood after $72-\mathrm{h}$ treatment with cytokine granulocyte colony-stimulating factor (G-CSF; three injections, $100 \mu \mathrm{g} / \mathrm{kg}$ per day) measured by qRT-PCR. Results were normalized to beta-2microglobulin (B2m) level. Data represent the mean value \pm SEM for four independent experiments. ${ }^{* *} p<0.01$; $* * * p<$ 0.001 (Student's $t$ test)
A

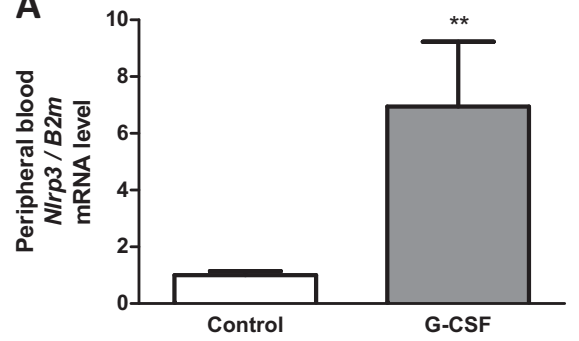

B

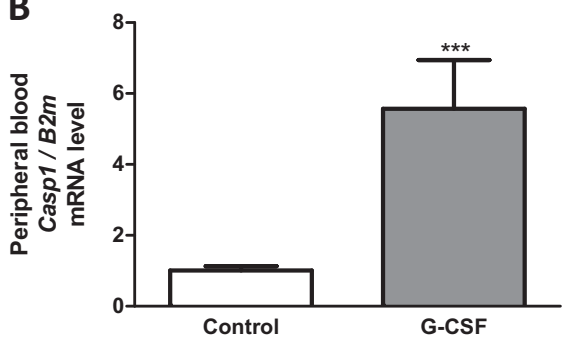

C

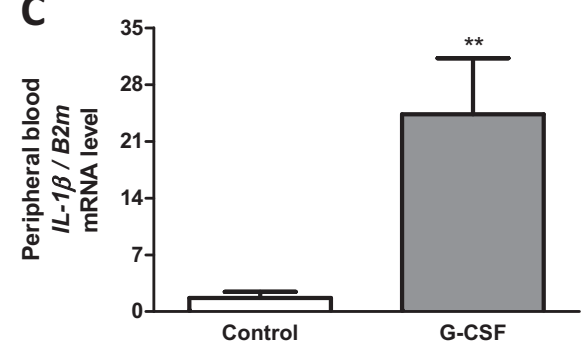

In the extracellular space, ATP is processed as a purinergic mediator by the cell surface-expressed ectonucleotidases CD39 and CD73 to its metabolites ADP and AMP (products of CD39) and adenosine (product of CD73) [35]. Of note, we reported that adenosine, in contrast to ATP, inhibits mobilization of HSPCs [19]. This occurs owing to adenosinemediated (i) upregulation of HO-1 and iNOS in HSPCs and granulocytes, which directly inhibits cell migration, (ii) direct inhibition of the inflammasome in innate immunity cells, and (iii) inhibition of the degranulation of granulocytes in the initiation phase of mobilization. Most importantly, adenosine activates the $\mathrm{P} 1$ family of $\mathrm{G}$ protein-coupled purinergic receptors (A1, A2A, A2B, and $\mathrm{A} 3$ ). As we have demonstrated, inhibition of the CD39 and CD73 ectonucleotidases, which process the degradation of ATP to adenosine in the extracellular space, enhances the mobilization of HSPCs [45]. Thus, as follow-up of this data we are currently investigating which of the P1 receptors is responsible for the mobilizationinhibitory effects of adenosine.

Figure 3 illustrates the general scheme of HSPC mobilization, depicting the promoting effect of ATP and the inhibitory effect of adenosine on the egress of HSPCs from $\mathrm{BM}$ into PB. It also shows the crucial involvement of $\mathrm{Gr}-1^{+}$ cell-released ATP in response to mobilizing agents on activation of the inflammasome and the release of several DAMPs and degranulation of neutrophils to release PLC32. DAMPs (Hmgb1 and S1009a) released during inflammasome activation trigger activation of the ComC and $\mathrm{CoaC}$ in an MBL-MASP-dependent manner. The scheme does not show the release of IL-1 $\beta$ and IL-18, which have a role in positive-feedback activation of the inflammasome.

In addition to hematopoietic cells, both EXNs and purinergic signaling modulate the function of other BM components, including mesenchymal cells and endothelial cells, and their impact on these cells during mobilization requires further study. Further work is also required to see whether other pro-mobilizing stimuli, such as growth-regulated protein beta [46], are also involved in purinergic signalinginitiated HSPC mobilization. It would be also interesting to see if inflammasomes in addition to HSPCs also affect mobilization of other BM-residing stem cells [47-49].

\section{Activation of the ComC and its importance for optimal release of HSPCs from BM in the execution phase of mobilization}

As mentioned above, mobilizing agents induce a cascade of events in the $\mathrm{BM}$ microenvironment that can be considered "sterile" inflammation [14, 19]. However, like microbialinduced inflammation, this state is marked by the activation of cellular and soluble elements of innate immunity, including neutrophils and macrophages, as well as the ComC. We have already reported the crucial role of the MBL-MASP pathway of ComC activation in the initiation phase of mobilization [26]. In further support of this finding, mice that are deficient in MBL or MASP-1 are poor mobilizers in response to G-CSF and AMD3100.

Next, ComC activation and release of C5 cleavage fragments has a crucial role in the execution phase of mobilization, and C5 is cleaved by classical C5 convertase, which is generated during activation of the proximal part of the ComC. In further support of this mechanism, we have demonstrated that mice deficient in the $\mathrm{C} 5$ component of the ComC are poor mobilizers in response to G-CSF and AMD3100 [50, 51]. In addition, "C5-like" convertase activity is also provided by thrombin as a result of $\mathrm{CoaC}$ activation [52]. Interestingly, inhibition of thrombin by refludan also has a negative effect on the release of HSPCs, 


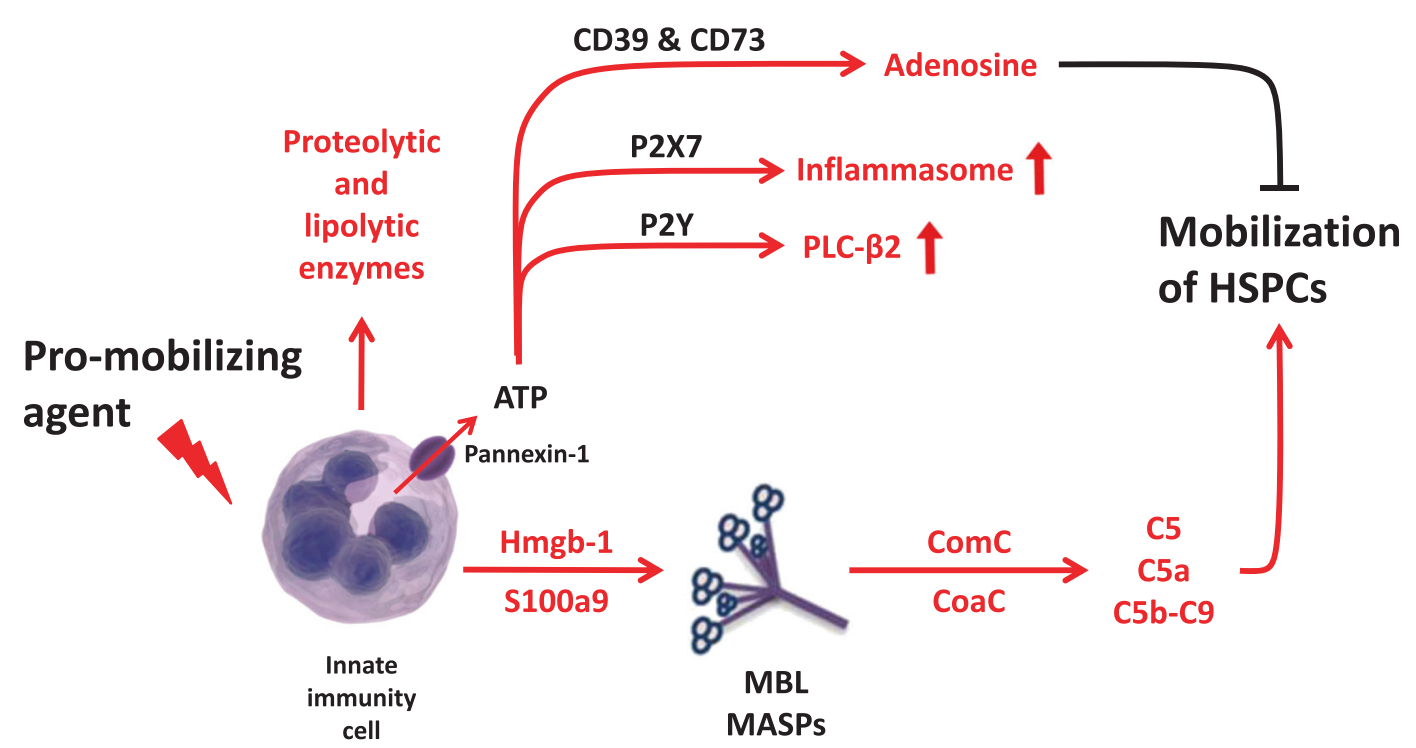

Initiation Phase

Fig. 3 The interplay between purinergic signaling and ComC activation during mobilization of HSPCs. Pro-mobilizing agents (e.g., GCSF) activate innate immunity cells (e.g., granulocytes or monocytes) to secrete proteolytic and lipolytic enzymes as well as several DAMPs, including ATP, Hmgb1, and S100a9. ATP is a potent activator of the inflammasome, which potentiates, through the $\mathrm{P} 2 \mathrm{X} 7$ receptor, the release of HMGB1 and S100a9 from innate immunity cells, and stimulates via $\mathrm{P} 2 \mathrm{Y}$ receptors the degranulation of neutrophils, which release more PLC- $\beta 2$ and proteolytic enzymes. In the next step, HGMB1 and S100a9 proteins activate the complement cascade $(\mathrm{ComC})$ in the MBL-dependent pathway, and PLC- $\beta 2$ disrupts lipid rafts on the surface of HSPCs, which play a role in the retention of HSPCs in BM stem cell niches. Thus, both DAMPs and PLC- $\beta 2$ promote effective mobilization. At the same time, ATP is processed to adenosine by CD39 and CD73 ectonucleotidases, which inhibits the mobilization process by (i) upregulating heme oxygenase 1 (HO-1)

which supports the existence of a proteolytic interplay between the two ancient proteolytic cascades [4, 12]. As it is shown in Fig. 3 inflammasome activates via MBL-MASP pathway both cascades.

Activation of the distal part of the ComC during the execution phase of mobilization and the resulting release of C5 cleavage fragments promotes egress of HSPCs by several mechanisms. The C5 cleavage fragments, C5a and desArg $\mathrm{C} 5 \mathrm{a}$, are generated both in the BM microenvironment and in BM-blood sinusoids [1, 50]. Primarily, they both activate innate immunity cells in the BM microenvironment by positive feedback and enhance the state of sterile inflammation. Secondarily, although C5 cleavage fragments alone do not chemoattract HSPCs, they are potent chemoattractants for granulocytes and monocytes [1, 37, 50]. Therefore, the $\mathrm{C} 5 \mathrm{a}$ and ${ }_{\text {desArg }} \mathrm{C} 5 \mathrm{a}$ present in $\mathrm{BM}$ sinusoids create a gradient across the BM-blood endothelial barrier for egress of granulocytes and monocytes, and these cells enriched for proteolytic enzymes pave the way for HSPCs to leave BM by "following in their footsteps". HSPCs then

\section{Amplification phase $\longrightarrow$ Execution phase}

and inducible nitric oxide synthetase (iNOS) in HSPCs and innate immunity cells and (ii) inhibiting the degranulation of neutrophils. The ComC, together with the CoaC, is activated in the MBL-dependent pathway, which releases $\mathrm{C} 5$ cleavage fragments, which are in turn crucial in inducing the egress of HSPCs from BM into PB. Pathways promoting mobilization are shown by red arrows and the adenosine inhibitory pathway by a black arrow. There are also indicated three phases of mobilization-initiation phase (activation of NRLP3 inflammasome and release of DAMPs activating the MBL pathway of ComC), amplification phase (NRLP3 products and alternative ComC activation pathway amplify sterile inflammation in $\mathrm{BM}$ ), and the execution phase (ComC cleavage fragments and activation of CoaC permeabilize BM-PB barrier to facilitate the egress of HSPCs). (adapted from Adamiak M. et al. Oncotarget, 2018, Vol. 9, (No. 90), pp: 36052-36054)

respond to the gradient of bioactive phosphosphingolipids (sphingosine-1-phosphate [S1P] and ceramide-1-phosphate [C1P]), which is already high, even under steady-state conditions in PB [1, 17, 53, 54]. Thus, after release from their niches HSPCs migrate to the BM sinusoids.

The important role of S1P and C1P in the egress of HSPCs is supported by the fact that both of these phosphosphingolipids create strong chemotactic gradients for HSPCs across the BM-PB endothelial barrier under steadystate conditions [17]. The fact that HSPCs released from their niches follow S1P and C1P gradients in PB indicates an active retention process for HSPCs in BM niches that counteracts these egress-promoting gradients. In support of this conclusion, the egress of HSPCs from BM is impaired in mice that have low levels of S1P in PB owing to sphingosine kinase 1 deficiency and, by contrast, is enhanced in mutant mice with sphingosine 2 kinase deficiency, which, surprisingly, have elevated levels of S1P in their PB plasma [17, 55]. Moreover, in addition to soluble C5a and desArg $\mathrm{C} 5 \mathrm{a}, \mathrm{C} 5$ cleavage leads to generation of 
membrane attack complex (MAC, also known as C5b-C9). MAC may additionally increase S1P levels in PB by inducing its release from erythrocytes, as these cells are highly enriched in this bioactive phosphosphingolipid [53].

Nevertheless, even strong upregulation of the S1P level in $\mathrm{PB}$, as seen for example during phenylohydrazineinduced hemolysis, will not mobilize HSPCs as long as they are anchored in BM niches. Robust mobilization occurs first after co-administration of AMD3100 [56], which indicates active retention of HSPCs in BM niches and the need to first decrease this retention of HSPCs in BM niches to enhance mobilization in response to S1P gradient [56].

\section{Novel potential strategies to enhance mobilization of HSPCs}

HSPCs mobilized into PB are easily isolated by leukapheresis, and they quickly engraft after transplantation. Thus, the pharmacological mobilization of HSPCs is a convenient strategy to obtain these cells for BM reconstitution after hematopoietic transplantation. Because some patients are poor mobilizers in response to conventional pro-mobilizing compounds (e.g., G-CSF), more efficient strategies are needed to obtain these cells for clinical purposes [1-5]. Based on the results presented in this review, it is reasonable to evaluate the efficacy of modulators of purinergic signaling, the inflammasome, and the ComC in enhancing this process.

As discussed, and as presented in Fig. 3, it is important to inhibit activity of the cell surface ectonucleotidases CD39 and CD73 to inhibit extracellular degradation of ATP to adenosine, which, as we have demonstrated, possesses antimobilizing properties $[19,45]$. There are small-molecule inhibitors of these enzymes available, and our preliminary results in a murine model indicates that they could be promising pro-mobilizing adjuvant compounds if employed with G-CSF or AMD3100 [45]. Taking into consideration the important role of the inflammasome, one could consider application of ATP as a therapy to activate the inflammasome [19]. Our preliminary results in a murine model confirmed that ATP, together with G-CSF or AMD3100, enhances mobilization efficacy [19]. It is also possible that other activators of the inflammasome, such as the antibiotic nigericin or other reagents opening potassium efflux channels [31], could find similar application as well. We are currently testing this possibility in animal models.

Other potential strategies to enhance mobilization are related to optimal and adequate activation of the ComC. As has been demonstrated by us and others, activation of the ComC is negatively regulated by HO- 1 and iNOS [40-44]. Therefore, small-molecule inhibitors of HO-1, as already shown in a murine model, could enhance mobilization of HSPCs if administered together with pro-mobilizing factors, as mentioned above [40]. This possibility is supported by

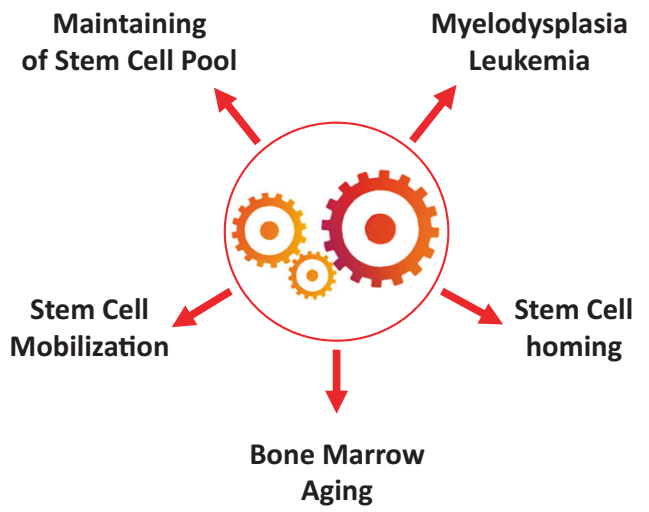

Fig. 4 The proposed involvement of the NRLP3 inflammasome in regulating hematopoiesis. The inflammasome (shown as interacting gears/cogwheels) couples purinergic signaling with the ComC, which may affect several processes in BM, including stem cell mobilization, stem cell homing and engraftment, maintaining the pool of HSPCs, regulating aging, and playing a role in myelodysplasia and, as a consequence, in the origin of leukemia

the fact that HO-1 and iNOS are potent inhibitors of NLRP3 inflammasome activation $[42,43]$.

\section{Future directions in studying the role of the NLRP3 inflammasome in normal hematopoiesis}

The potential pleiotropic effects of the NLRP3 inflammasome on hematopoiesis are depicted in Fig. 4. In addition to the role of the NLRP3 inflammasome in the mobilization of HSPCs, further studies are needed to see whether it is also involved in the homing of HSPCs to BM. To justify this concept, we have demonstrated in the past that activation of the ComC in the BM microenvironment after conditioning for transplantation may have a role in accelerating hematopoietic reconstitution after HSPC transplantation [57]. Providing further support for this concept, by employing the Transwell system we demonstrated that, in addition to SDF1 , S1P, and C1P, ATP is a chemoattractant for HSPCs and thus a potential candidate homing factor for HSPCs [58].

To assess the role of ATP in homing and engraftment, we transplanted HSPCs from CXCR4 $4^{\mathrm{f} / \mathrm{fl}} \mathrm{Cre}^{\mathrm{Tg} /-}$ mice, in which CXCR4 had been eliminated by a Cre hematopoietic driver strategy into sphingosine kinase 1-deficient $\left(\mathrm{Sphk}^{-{ }^{-}}\right)$mice, which are deficient for S1P expression in BM. These experiments in mice, based on two defective homing axes, SDF-1-CXCR4 and S1P-S1P1 receptor, demonstrated the involvement of still other factor/s in the homing process, and ATP became the most likely candidate [58]. Specifically, ATP is released from cells in the BM microenvironment during conditioning for transplantation, and in addition to SDF-1 and S1P, could be involved as a homing chemoattractant for HSPCs. We also recently observed that exposure of HSPCs to a small-molecule inflammasome 
inhibitor before transplantation into lethally irradiated mice affects their homing and engraftment. Thus, the ATPstimulated inflammasome has here a significant role (manuscript in preparation). In further support for a role of the inflammasome in HSPC homing and engraftment, our most recent results revealed that HSPCs exposed before transplantation to a $\mathrm{P} 2 \mathrm{X} 7$ receptor antagonist, which prevents ATP-mediated activation of the inflammasome, also engraft more poorly than normal HSPCs.

A breakthrough in NLRP3 inflammasome research came from the observation that inflammasomes can also be released by macrophages as extracellular oligomeric particulate complexes. These particulate complexes may activate caspase 1 in the extracellular milieu, amplify inflammation, and be internalized by bystander cells and thereby contribute to the spread of the inflammatory reaction to these cells [21-23]. Moreover, we postulate that this phenomenon could involve extracellular microvesicles [59]. How important this intriguing phenomenon is in HSPC mobilization requires further study.

Another important aspect to investigate is the potential role of ATP-mediated inflammasome activation in regulating the pool of HSPCs via IL-1 $\beta$. Inflammasome-mediated IL- $1 \beta$ signaling appears to serve as an integrator of metabolic activity downstream of ROS-HIF1 $\alpha$ to promote HSPC formation and the development of the myeloid and lymphoid lineages in vivo and in vitro [60]. ATP that is secreted by cells in the BM microenvironment could have a role as an NLRP3 inflammasome activator to regulate IL-1 $\beta$ expression and thus have a role in regulating the pool of HSPCs.

Another important approach is to study the role of the inflammasome directly in hematopoietic niches. As of today, the hematopoietic stem cell niche remains incompletely defined and is described by competing models. This niche most likely is perivascular $\left(\mathrm{SDF}-1^{+}\right.$and $\left.\mathrm{KL}^{+}\right)$, created partially by mesenchymal stromal cells and endothelial cells, and is often, but not always, located near trabecular bone in the BM microenvironment [5, 61, 62]. Although HSCs are located around perivascular cells, early lymphoid progenitors are associated with the osteoblastic niche. The effect of ATP-inflammasome signaling in maintaining the integrity of these niches under steady-state conditions and during mobilization requires further study. Such investigations may also be relevant to better understanding the process of HSPC aging and the pathogenesis of myelodysplastic syndromes, in which the NRLP3 inflammasome has a role [63, 64].

Finally, after crossing a threshold of activation, the NRLP3 inflammasome may induce a form of cell death known as pyroptosis [21-23]. In this context, there are other members of this NLRP protein family, such as NLRP2, NLRPC3, NLRP6, NLRP7, NLRP10, NLRP12, and
NLRX1, which have been suggested as playing inhibitory roles during inflammation by controlling caspase 1mediated IL- $1 \beta$ secretion or by suppressing NF- $\kappa$ B signaling [21-23]. It would be interesting if these particular inflammasomes inhibit the effects of other proinflammatory inflammasomes in preventing stem cell mobilization, aging, and myelodysplasia

\section{Conclusions}

We claim that innate immunity-mediated sterile inflammation induced in the $\mathrm{BM}$ microenvironment by promobilization stimuli activates release of ATP from innate immunity cells. By involving P2X7 receptors, this release activates the NLRP3 inflammasome, which has the role of a "cogwheel" coupling purinergic signaling with activation of the ComC. This coupling is necessary to ensure optimal release of HSPCs from BM. In support of this notion, the NLRP3 inflammasome becomes activated during mobilization of HSPCs, and inhibition of its crucial component ASC results in a decrease in this process. Beside its role in mobilization of HSPCs, the NLRP3 inflammasome is also involved in maintaining the pool of HSPCs in BM niches. We are currently studying its role in the homing and engraftment of HSPCs after transplantation. As it has been reported that, besides the NRLP3 inflammasome, the NLRP1 and NLRP12 inflammasomes are expressed by hematopoietic cells, further study is needed to address whether they are also involved in HSPC trafficking. In support of this possibility, ATP activates the NLRP1 inflammasome by interacting with the $\mathrm{P} 2 \mathrm{X} 4$ receptor in some cells. Moreover, some recent data indicate that NLRC4 and AIM2 inflammasomes are may be also activated be factors released during "sterile" inflammation [29] and their potential involvement in HSPCs mobilization clarification. Finally, further work should also address the role of NLRP family members (NLRP2, NLRC3, NLRP6, NLRP7, NLRP10, NLRP12, and NLRX1) in inhibiting inflammation [21-23, 27-29, 65], as they could have a negative role in the release of HSPCs from BM.

Acknowledgements This work was supported by NIH grants 2R01 DK074720 and R01HL112788, the Stella and Henry Endowment, and the OPUS grant UMO-2018/29/B/NZ4/01470 to MZR. AT was supported by NIH T32 HL134644 to MZR

\section{Compliance with ethical standards}

Conflict of interest The authors declare that they have no conflict of interest.

Publisher's note: Springer Nature remains neutral with regard to jurisdictional claims in published maps and institutional affiliations. 
Open Access This article is licensed under a Creative Commons Attribution 4.0 International License, which permits use, sharing, adaptation, distribution and reproduction in any medium or format, as long as you give appropriate credit to the original author(s) and the source, provide a link to the Creative Commons license, and indicate if changes were made. The images or other third party material in this article are included in the article's Creative Commons license, unless indicated otherwise in a credit line to the material. If material is not included in the article's Creative Commons license and your intended use is not permitted by statutory regulation or exceeds the permitted use, you will need to obtain permission directly from the copyright holder. To view a copy of this license, visit http://creativecommons. org/licenses/by/4.0/.

\section{References}

1. Ratajczak MZ. A novel view of the adult bone marrow stem cell hierarchy and stem cell trafficking. Leukemia. 2015;29:776-82.

2. Hoggatt J, Pelus LM. Many mechanisms mediating mobilization: an alliterative review. Curr Opin Hematol. 2011;18:231-8.

3. Schuettpelz LG, Link DC. Regulation of hematopoietic stem cell activity by inflammation. Front Immunol. 2013;4:204.

4. Nguyen TS, Lapidot T, Ruf W. Extravascular coagulation in hematopoietic stem and progenitor cell regulation. Blood. 2018;132:123-31.

5. Tay J, Levesque JP, Winkler IG. Cellular players of hematopoietic stem cell mobilization in the bone marrow niche. Int $\mathrm{J}$ Hematol. 2017;105:129-40.

6. Massberg S, Schaerli P, Knezevic-Maramica I, Köllnberger M, Tubo N, Moseman EA, et al. Immunosurveillance by hematopoietic progenitor cells trafficking through blood, lymph, and peripheral tissues. Cell. 2007;131:994-1008.

7. Levesque JP, Helwani FM, Winkler IG. The endosteal 'osteoblastic' niche and its role in hematopoietic stem cell homing and mobilization. Leukemia. 2010;24:1979-92.

8. Mendez-Ferrer S, Chow A, Merad M, Frenette PS. Circadian rhythms influence hematopoietic stem cells. Curr Opin Hematol. 2009;16:235-42.

9. Borkowska S, Suszynska M, Ratajczak J, Ratajczak MZ. Evidence of a pivotal role for the distal part of the complement cascade in the diurnal release of hematopoietic stem cells into peripheral blood. Cell Transplant. 2016;2:275-82.

10. Itkin T, Kumari A, Schneider E, Gur-Cohen S, Ludwig C, Brooks $\mathrm{R}$, et al. MicroRNA-155 promotes G-CSF-induced mobilization of murine hematopoietic stem and progenitor cells via propagation of CXCL12 signaling. Leukemia. 2017;31:1247-50.

11. Ratajczak MZ, Adamiak M. Membrane lipid rafts, master regulators of hematopoietic stem cell retention in bone marrow and their trafficking. Leukemia. 2015;7:1452-7.

12. Borkowska S, Suszynska M, Mierzejewska K, Ismail A, Budkowska M, Salata D, et al. Novel evidence that crosstalk between the complement, coagulation and fibrinolysis proteolytic cascades is involved in mobilization of hematopoietic stem/progenitor cells (HSPCs). Leukemia. 2014;28:2148-54.

13. Ratajczak MZ, Kim CH, Wojakowski W, Janowska-Wieczorek A, Kucia M, Ratajczak J. Innate immunity as orchestrator of stem cell mobilization. Leukemia. 2010;24:1667-75.

14. Ratajczak MZ, Adamiak M, Plonka M, Abdel-Latif A, Ratajczak J. Mobilization of hematopoietic stem cells as a result of innate immunity-mediated sterile inflammation in the bone marrow microenvironment-the involvement of extracellular nucleotides and purinergic signaling. Leukemia. 2018;32:1116-23.

15. Chen GY, Nuñez G. Sterile inflammation: sensing and reacting to damage. Nat Rev Immunol. 2010;10:826-37.
16. Winkler IG, Pettit AR, Raggatt LJ, Jacobsen RN, Forristal CE, Barbier V, et al. Hematopoietic stem cell mobilizing agents GCSF, cyclophosphamide or AMD3100 have distinct mechanisms of action on bone marrow HSC niches and bone formation. Leukemia. 2012;26:1594-601.

17. Golan K, Vagima Y, Ludin A, Itkin T, Cohen-Gur S, Kalinkovich A, et al. S1P promotes murine progenitor cell egress and mobilization via S1P1-mediated ROS signaling and SDF-1 release. Blood. 2012;119:2478-88.

18. Adamiak M, Poniewierska-Baran A, Borkowska S, Schneider G, Abdelbaset-Ismail A, Suszynska M, et al. Evidence that a lipolytic enzyme-hematopoietic-specific phospholipase C- $\beta 2$ - promotes mobilization of hematopoietic stem cells by decreasing their lipid raft-mediated bone marrow retention and increasing the promobilizing effects of granulocytes. Leukemia. 2016;30:919-28.

19. Adamiak M, Bujko K, Cymer M, Plonka M, Glaser T, Kucia M, et al. Novel evidence that extracellular nucleotides and purinergic signaling induce innate immunity-mediated mobilization of hematopoietic stem/progenitor cells. Leukemia. 2018;32:1920-31.

20. Lemoli RM, Ferrari D, Fogli M, Rossi L, Pizzirani C, Forchap S, et al. Extracellular nucleotides are potent stimulators of human hematopoietic stem cells in vitro and in vivo. Blood. 2004;104:1662-70.

21. Groslambert M, Py BF. Spotlight on the NLRP3 inflammasome pathway. J Inflamm Res. 2018;11:359-74.

22. He Y, Hara H, Núñez G. Mechanism and regulation of NLRP3 inflammasome activation. Trends Biochem Sci. 2016;41:1012-21.

23. Place DE, Kanneganti TD. Recent advances in inflammasome biology. Curr Opin Immunol. 2018;50:32-38.

24. Malik A, Kanneganti TD. Function and regulation of IL- $1 \alpha$ in inflammatory diseases and cancer. Immunol Rev. 2018;281: 124-37.

25. Fibbe WE, Hamilton MS, Laterveer LL, Kibbelaar RE, Falkenburg JH, Visser JW, et al. Sustained engraftment of mice transplanted with IL-1-primed blood-derived stem cells. J Immunol. 1992;148:417-21.

26. Adamiak M, Abdelbaset-Ismail A, Suszynska M, Abdel-Latif A, Ratajczak J, Ratajczak MZ. Novel evidence that the mannanbinding lectin pathway of complement activation plays a pivotal role in triggering mobilization of hematopoietic stem/progenitor cells by activation of both the complement and coagulation cascades. Leukemia. 2017;31:262-5.

27. Masters SL, Gerlic M, Metcalf D, Preston S, Pellegrini M, O'Donnell JA, et al. NLRP1 inflammasome activation induces pyroptosis of hematopoietic progenitor cells. Immunity. 2012;37:1009-23.

28. Tuncer S, Fiorillo MT, Sorrentino R. The multifaceted nature of NLRP12. J Leukoc Biol. 2014;96:991-1000.

29. Denes A, Coutts G, Lénárt N, Cruickshank SM, Pelegrin P, Skinner J, et al. AIM2 and NLRC4 inflammasomes contribute with ASC to acute brain injury independently of NLRP3. Proc Natl Acad Sci USA. 2015;112:4050-5.

30. Marquez-Curtis LA, Turner AR, Sridharan S, Ratajczak MZ, Janowska-Wieczorek A. The ins and outs of hematopoietic stem cells: studies to improve transplantation outcomes. Stem Cell Rev. 2011;3:590-607.

31. Di A, Xiong S, Ye Z, Malireddi RKS, Kometani S, Zhong M, et al. The TWIK2 potassium efflux channel in macrophages mediates NLRP3 inflammasome-Induced inflammation. Immunity. 2018;49:56-65.e4.

32. Velders GA, van Os R, Hagoort H, Verzaal P, Guiot HF, Lindley IJ, et al. Reduced stem cell mobilization in mice receiving antibiotic modulation of the intestinal flora: involvement of endotoxins as cofactors in mobilization. Blood. 2004;103:340-6.

33. Schuettpelz LG, Borgerding JN, Christopher MJ, Gopalan PK, Romine MP, Herman AC, et al. G-CSF regulates hematopoietic 
stem cell activity, in part, through activation of Toll-like receptor signaling. Leukemia. 2014;28:1851-60.

34. Gonzalez-Nieto D, Li L, Kohler A, Ghiaur G, Ishikawa E, Sengupta A, et al. Connexin-43 in the osteogenic BM niche regulates its cellular composition and the bidirectional traffic of hematopoietic stem cells and progenitors. Blood. 2012;119:5144-54.

35. Burnstock G. An introduction to the roles of purinergic signalling in neurodegeneration, neuroprotection and neuroregeneration. Neuropharmacology. 2016;104:4-17.

36. Koldej R, Collins J, Ritchie D. P2X7 polymorphisms and stem cell mobilisation. Leukemia. 2018;32:2724-6.

37. Junger WG. Immune cell regulation by autocrine purinergic signalling. Nat Rev Immunol. 2011;11:201-12.

38. Pruijt JF, Verzaal P, Van Os R, de Kruijf EJ, van Schie ML, Mantovani A, et al. Neutrophils are indispensable for hematopoietic stem cell mobilization induced by interleukin-8 in mice. Proc Natl Acad Sci USA. 2002;99:6228-33.

39. Levesque JP, Hendy J, Takamatsu Y, Williams B, Winkler IG, Simmons PJ. Mobilization by either cyclophosphamide or granulocyte colony-stimulating factor transforms the bone marrow into a highly proteolytic environment. Exp Hematol. 2002;30:440-9.

40. Wysoczynski M, Ratajczak J, Pedziwiatr D, Rokosh G, Bolli R, Ratajczak MZ. Identification of heme oxygenase 1 (HO-1) as a novel negative regulator of mobilization of hematopoietic stem/ progenitor cells. Stem Cell Rev. 2015;11:110-8.

41. Adamiak M, Abdelbaset-Ismail A, Moore JB 4th, Zhao J, AbdelLatif A, Wysoczynski M, et al. Inducible nitric oxide synthase (iNOS) is a novel negative regulator of hematopoietic stem/progenitor cell trafficking. Stem Cell Rev. 2017;13:92-103.

42. Nurmi K, Kareinen I, Virkanen J, Rajamäki K, Kouri VP, Vaali K, et al. Hemin and Cobalt protoporphyrin inhibit NLRP3 inflammasome activation by enhancing autophagy: a novel mechanism of inflammasome regulation. J Innate Immun. 2017;9:65-82.

43. Hernandez-Cuellar E, Tsuchiya K, Hara H, Fang R, Sakai S, Kawamura I, et al. Cutting edge: nitric oxide inhibits the NLRP3 inflammasome. J Immunol. 2012;189:5113-7.

44. Abdelbaset-Ismail A, Borkowska-Rzeszotek S, Kubis E, Bujko K, Brzeźniakiewicz-Janus K, Bolkun L, et al. Activation of the complement cascade enhances motility of leukemic cells by downregulating expression of HO-1. Leukemia. 2017;31:446-58.

45. Adamiak M, Bujko K, Lenkiewicz A, Kucia M, Ratajczak J, Ratajczak MZ. Novel evidence that the ectonucleotidases CD39 and CD73, which are expressed on hematopoietic stem/progenitor cells (HSPCs), regulate mobilization and homing - studies in CD39-/- and CD73-/- mice and with small-molecule CD39 and CD73 inhibitors. Blood. 2018;132:2060.

46. Hoggatt J, Singh P, Tate TA, Chou BK, Datari SR, Fukuda S, et al. Rapid mobilization reveals a highly engraftable hematopoietic stem cell. Cell. 2018;172:191-204.e10.

47. Ratajczak MZ, Bartke A, Darzynkiewicz Z. Prolonged growth hormone/insulin/insulin-like growth factor nutrient response signaling pathway as a silent killer of stem cells and a culprit in aging. Stem Cell Rev. 2017;13:443-53.

48. Bhartiya D. Pluripotent stem cells in adult tissues: struggling to be acknowledged over two decades. Stem Cell Rev. 2017;13:713-24.

49. Smadja DM. Bone marrow very small embryonic-like stem cells: new generation of autologous cell therapy soon ready for prime time? Stem Cell Rev. 2017;13:198-201.

50. Lee HM, Wysoczynski M, Liu R, Shin DM, Kucia M, Botto M, et al. Mobilization studies in complement-deficient mice reveal that optimal AMD3100 mobilization of hematopoietic stem cells depends on complement cascade activation by AMD3100stimulated granulocytes. Leukemia. 2010;24:573-82.
51. Lee HM, Wu W, Wysoczynski M, Liu R, Zuba-Surma EK, Kucia M, et al. Impaired mobilization of hematopoietic stem/ progenitor cells in C5-deficient mice supports the pivotal involvement of innate immunity in this process and reveals novel promobilization effects of granulocytes. Leukemia. 2009;23:2052-62.

52. Huber-Lang M, Sarma JV, Zetoune FS, Rittirsch D, Neff TA, McGuire SR, et al. Generation of C5a in the absence of C3: a new complement activation pathway. Nat Med. 2006;12:682-7.

53. Ratajczak MZ, Lee H, Wysoczynski M, Wan W, Marlicz W, Laughlin MJ, et al. Novel insight into stem cell mobilizationplasma sphingosine-1-phosphate is a major chemoattractant that directs the egress of hematopoietic stem progenitor cells from the bone marrow and its level in peripheral blood increases during mobilization due to activation of complement cascade/membrane attack complex. Leukemia. 2010;24:976-85.

54. Juarez JG, Harun N, Thien M, Welschinger R, Baraz R, Pena AD, et al. Sphingosine-1-phosphate facilitates trafficking of hematopoietic stem cells and their mobilization by CXCR4 antagonists in mice. Blood. 2012;119:707-16.

55. Adamiak M, Chelvarajan L, Lynch KR, Santos WL, Abdel-Latif A, Ratajczak MZ. Mobilization studies in $m$ ice deficient in sphingosine kinase 2 support a crucial role of the plasma level of sphingosine-1-phosphate in the egress of hematopoietic stem progenitor cells. Oncotarget. 2017;39:65588-600.

56. Mierzejewska K, Klyachkin YM, Ratajczak J, Abdel-Latif A, Kucia M, Ratajczak MZ. Sphingosine-1-phosphate-mediated mobilization of hematopoietic stem/progenitor cells during intravascular hemolysis requires attenuation of SDF-1-CXCR4 retention signaling in bone marrow. Biomed Res Int. 2013;2013:814549.

57. Kim $\mathrm{CH}, \mathrm{Wu} \mathrm{W}$, Wysoczynski M, Abdel-Latif A, Sunkara M, Morris A, et al. Conditioning for hematopoietic transplantation activates the complement cascade and induces a proteolytic environment in bone marrow: a novel role for bioactive lipids and soluble C5b-C9 as homing factors. Leukemia. 2012;26:106-16.

58. Adamiak M, Borkowska S, Wysoczynski M, Suszynska M, Kucia M, Rokosh G, et al. Evidence for the involvement of sphingosine1-phosphate in the homing and engraftment of hematopoietic stem cells to bone marrow. Oncotarget. 2015;6:18819-28.

59. Cypryk W, Nyman TA, Matikainen S. From inflammasome to exosome-does extracellular vesicle secretion constitute an inflammasome-dependent immune response? Front Immunol. 2018;9:2188.

60. Frame JM, Long T, Schuster-Kubaczka C, Esain V, Lim SE, Daley GQ, et al. Inflammasome-mediated regulation of hematopoiesis in the vertebrate embryo. Blood. 2018;132:330.

61. Baryawno N, Severe N, Scadden DT. Hematopoiesis: reconciling historic controversies about the niche. Cell Stem Cell. 2017;5:590-2.

62. Mendelson A, Frenette PS. Hematopoietic stem cell niche maintenance during homeostasis and regeneration. Nat Med. 2014;8:833-46.

63. Sallman DA, Cluzeau T, Basiorka AA, List A. Unraveling the pathogenesis of MDS: the NLRP3 inflammasome and pyroptosis drive the MDS phenotype. Front Oncol. 2016;6:151.

64. Kovtonyuk LV, Fritsch K, Feng X, Manz MG, Takizawa H. Inflamm-aging of hematopoiesis, hematopoietic stem cells, and the bone marrow microenvironment. Front Immunol. 2016; 7:502.

65. Linz BM, Neely CJ, Kartchner LB, Mendoza AE, Khoury AL, Truax A, et al. Innate immune cell recovery is positively regulated by NLRP12 during emergency hematopoiesis. J Immunol. 2017;198:2426-33. 